\title{
Conexões
}

\section{Tai Chi Chuan em posição sentada: uma revisão sistemática}

\author{
Samuel Ribeiro dos Santos Neto ${ }^{1}$ \\ Hélio Mamoru Yoshida ${ }^{1}$ \\ Igor Cavalcante $\mathrm{Doi}^{1}$
}

\section{RESUMO}

O presente trabalho teve por objetivo realizar uma revisão sistemática da literatura científica sobre Tai Chi Chuan em posição sentada, entre 2007 e 2017. Foram analisados 17 artigos em língua inglesa e portuguesa. Os trabalhos foram divididos em quatro aspectos: estudos cinemáticos, abordagens político-práticas, aspectos físicos e aspectos psicológicos. A revisão concluiu que as pesquisas sobre Tai Chi em posição sentada centram-se no aspecto terapêutico, ligado ao paradigma da saúde, e boa parte delas carece de precisão metodológica e de amostras populacionais mais amplas. No geral, indicou-se que a prática, de baixo custo e acessível, pode promover melhoras em parâmetros físicos, como equilíbrio postural e amplitude de movimento de ombros, e psicológicos, como autoestima, autoeficácia e qualidade de vida. Além disso, as técnicas do Tai Chi Chuan podem ser facilmente adaptáveis a pessoas com diferentes graus de prejuízo motor. A escassez de estudos sobre o tema sugere a necessidade de novas e mais precisas pesquisas.

Palavras-chave: Tai Ji. Pessoas com deficiência. Revisão.

\footnotetext{
${ }^{1}$ Universidade Estadual de Campinas Recebido em: 7 maio 2018

Aprovado em: 2 out. 2018

Contato: samuca_1990@yahoo.com.br
} 


\section{Sitting Tai Chi Chuan: a systematic review}

\section{Abstract}

The aim of this work was to carry out a systematic review of the scientific literature on Tai Chi Chuan in a seated position between 2007 and 2017. Seventeen articles were analyzed in English and Portuguese. The works were divided into four aspects: kinematic studies, political-practical approaches, physical aspects and psychological aspects. The review concluded that research on Tai Chi in a seated position focuses on the therapeutic aspect, linked to the health paradigm, and much of it lacks methodological accuracy and the use of broader population samples. In general, it was indicated that the practice, with low cost and accessible, can promote improvements in physical parameters such as postural balance and range of shoulder movement, and psychological, such as selfesteem, self-efficacy and quality of life. In addition, Tai Chi Chuan techniques can be easily adaptable to people with varying degrees of motor impairment. The scarcity of studies on the subject suggest the need for new and more precise research.

Keywords: Tai Ji. Disabled persons. Review.

\section{Tai Chi Chuan en posición sentada: una revisión sistemática}

\section{RESUMEN}

Este estudio tuvo como objetivo llevar a cabo una revisión sistemática de la literatura científica sobre el Tai Chi Chuan en posición sentada entre 2007 y 2017. Se analizaron 17 artículos en Inglés y portugués. Los trabajos se dividieron en cuatro aspectos: estudios cinemáticos, enfoques políticoprácticos, aspectos físicos y aspectos psicológicos. La revisión concluyó que las investigaciones sobre Tai Chi en posición sentada se centran en el aspecto terapéutico, ligado al paradigma de la salud, y buena parte de ellas carece de precisión metodológica y de muestras poblacionales más amplias. En general, se indicó que la práctica, barata y accesible, puede promover mejoras en parámetros físicos, como equilibrio postural y amplitud de movimiento de hombros, y psicológicos, como autoestima, autoeficacia y calidad de vida. Además, las técnicas del Tai Chi Chuan pueden ser fácilmente adaptables a las personas con diferentes grados de prejuicio motor. La escasez de estudios sobre el tema sugiere la necesidad de nuevas y más precisas investigaciones.

Palabras Clave: Tai Ji. Personas con Discapacidad. Revisión. 


\section{INTRODUÇÃO}

As últimas décadas viram crescer a procura da prática de atividades físicas por pessoas com deficiência. No Brasil, nos anos 1980 o Ministério da Educação e Cultura já se manifestava sobre o tema, enquanto surgia, na mesma década, o periódico científico internacional Adapted Physical Activity Querterly (BROADHEAD, 2017). A existência de associações que incentivam pesquisa nessa área, como a International Federation of Adapted Physical Activity, também aponta para o avanço nos interesses científicos e sociais nesse tema. Além daquela, a North American Federation of Adapted Physical Activity (NAFAPA) e a Sociedade Brasileira de Atividade Motora Adaptada (Sobama) são outros exemplos de organizações que têm promovido eventos acadêmicos desde os anos 1990 (NAFAPA, 2016; SOUZA, 2012), década que presenciou o aparecimento de periódicos científicos brasileiros como o Brasilian International Journal of Adapted Physical Activity Research e a Revista da Associação Brasileira de Atividade Motora Adaptada (SILVA; NETO; DRIGO, 2012).

Os diversos campos ligados ao treinamento e à Educação Física têm se preocupado com esse tema e avançado do ponto de vista da oferta de serviços e do estudo de práticas de lazer físico-esportivas (e.g.: MUNSTER; ALMEIDA, 2016; MELO; FUMES, 2013), de acessibilidade em ambientes de lazer físico-esportivos (e.g.: SANTOS et al., 2017; SILVA et al., 2013) e de treinamento esportivo (e.g.: MARTIN; WHALEN, 2014), além de discussões pedagógicas (e.g.: PALMA et al., 2012; ROSWAL; SHERRILL; ROSWAL, 1988). A popularização da questão das atividades físicas voltadas para essa população não se restringe ao campo dos esportes, mas está presente, por exemplo, no âmbito da dança (e.g.: ROSSI; MUNSTER, 2013; FIGUEIREDO; TAVARES, VENÂNCIO, 1999; GOODWIN; KROHN; KUHNLE, 2004) e de diversas outras práticas corporais, inclusive aquelas conhecidas como terapias alternativas (Ioga, Lian Gong, Tai Chi Chuan).

Em se tratando especificamente do Tai Chi Chuan ${ }^{2}$, convencionalmente feito em pé e com deslocamentos espaciais, é possível notar um grau de adaptabilidade em suas técnicas que o permitem ser praticado sob diferentes condições. Em vista disso, este artigo pretende explorar a produção científica sobre as adaptações do Tai Chi Chuan. Trata-se de uma apropriação contemporânea de uma prática corporal que é centenária, ligada à filosofia taoísta e à medicina tradicional chinesa.

O Tai Chi Chuan é uma arte marcial oriunda da China, cujo surgimento remonta ao século XVII. Caracteriza-se pela execução predominantemente lenta dos movimentos, cada qual representando um ataque ou defesa em uma luta com um oponente imaginário. A sequência coreografada de movimentos chama-se forma ou taolu. Formalmente, o Tai Chi é um estilo de Wushu - nome dado às diferentes artes marciais chinesas, pouco exploradas na literatura científica ocidental (YOSHIDA; SANTOS NETO, 2016) - embora seja

\footnotetext{
${ }^{2}$ A palavra Tai Chi Chuan pode ser grafada de muitas maneiras. No decorrer do texto, utilizamos a forma mais popularmente conhecida. Em nossas palavras-chave recorremos ao uso do termo Tai Ji, por tratar-se da forma utilizada nos Descritores em Ciências da Saúde (DeCS).
} 
frequentemente apartado desse conjunto no imaginário popular devido às suas particularidades.

Outrora praticado por círculos sociais restritos, o Tai Chi Chuan foi disseminado como atividade de saúde por meio de políticas da República Popular da China, a partir dos anos 1950, tendo gradualmente se popularizado em outros países ao longo do tempo. Importante frisar que esse processo de sistematização se inicia já no início do século XX, anteriormente à Revolução Comunista, quando surgem associações para a difusão das artes marciais tradicionais chinesas, e se estende após a revolução a partir da entrada do Wushu nas universidades e na pauta dos projetos esportivos oficiais do governo (ANTUNES, 2016).

Atualmente, o Tai Chi tem sido adotado com diferentes enfoques. Tanto seus estilos tradicionais como suas interpretações contemporâneas, habitualmente chamadas de modernas, são praticados como modalidades esportivas oficiais da International Wushu Federation (IWUF). Há também, notadamente na Ásia, escolas e grupos mais ligados às aplicações marciais dos movimentos, encarando a prática como forma de combate. Além disso, um modo bastante difundido de se praticar o Tai Chi Chuan é aquele que o encara como atividade meditativa, voltada à saúde e qualidade de vida através dos movimentos, executados preferencialmente em grupo e ao ar livre. Essa talvez seja sua expressão mais presente no imaginário coletivo, habitando cenas de filmes e reportagens, frequentemente associada à presença de idosos.

A prática, lenta e com baixo impacto, ganhou espaço em pesquisas e revisões devido à sua viabilidade de ser feita por perfis heterogêneos de pessoas. Quase sempre, o Tai Chi como objeto de pesquisa vem associado a temas ligados à saúde, qualidade de vida e reabilitação.

Em análise bibliométrica realizada por Yang et al. (2015), foram levantados 507 estudos clínicos envolvendo o Tai Chi Chuan, entre 1958 e 2013, sendo que praticamente metade deles, 255, tratavam-se de revisões sistemáticas. No conjunto levantado, diversas doenças e agravos foram analisados a partir de intervenções com Tai Chi, destacando-se a hipertensão, diabetes, osteoartrite, osteoporose/osteopenia, câncer de mama, insuficiência cardíaca, doença pulmonar obstrutiva crônica, doença arterial coronária, esquizofrenia e depressão.

Devido ao grande volume e à heterogeneidade da produção sobre o tema, não será o objetivo aqui levantar todas as hipóteses e resultados. Contudo, convém citar as conclusões das principais revisões sistemáticas publicadas nos últimos anos, a título de contextualizar o debate que pretendemos fazer.

De modo geral, as últimas pesquisas publicadas associaram as intervenções clínicas que fizeram uso do Tai Chi Chuan à melhora da qualidade do sono em pessoas idosas (DU et al., 2015), à prevenção primária de derrames em adultos de meia idade e idosos (ZHENG et al., 2015a), ao efeito positivo no treinamento cardiorrespiratório (ZHENG et al., 2015b) e na proteção da habilidade cognitiva (ZHENG et al., 2015c) em adultos saudáveis. Uma revisão 
brasileira também associou o Tai Chi a efeitos positivos na reabilitação cardíaca de pacientes com doença arterial coronária (NERY et al., 2014). A prática também parece auxiliar na reabilitação das funções motoras dos membros superiores de pacientes sobreviventes de câncer de mama (PAN et al., 2015).

Por outro lado, em revisão de Lee et al. (2015) não foi identificada qualquer relação positiva entre a intervenção com o Tai Chi Chuan e o tratamento de diabetes tipo 2. Quanto ao tratamento de dores crônicas, embora a revisão de Kong et al. (2016) tenha apontado que a prática pode ser um recurso complementar eficiente, Hall et al. (2017) concluíram que as pesquisas clínicas com Tai Chi carecem de rigor metodológico e que, portanto, não oferecem indícios suficientes que provem sua eficácia no quesito.

Do ponto de vista psicológico, associou-se a prática à melhora do bem estar psicológico, bem como a efeitos positivos sobre quadros de depressão e ansiedade (WANG et al., 2014). Outras melhoras também foram observadas em revisões, tais como sobre o medo de quedas em idosos (MARINHO et al., 2007) e sobre sua autoimagem e autoestima (GUIMARÃES et al., 2018).

Boa parte dessas revisões e das pesquisas clínicas às quais remontam dizem respeito à prática convencional do Tai Chi Chuan, destinada a pessoas sem deficiências de qualquer tipo, seja em situação típica de funcionalidade em suas vidas ou em situações de reabilitação em decorrência de algum agravo ou do próprio processo de envelhecimento. Portanto, tratamse de intervenções que não podem, sem adaptações, ser aplicadas sobre uma população com deficiência visual ou usuária de cadeira de rodas, por exemplo.

Na presente revisão, pretendemos abordar especificamente o Tai Chi Chuan feito em posição sentada, em cadeira de rodas ou cadeira convencional, podendo ou não ter movimentação nas articulações dos quadris, joelhos e tornozelos. Assim, esperamos olhar para formas adaptadas da prática que contemplem pessoas com lesão medular, com amputações de membros inferiores ou, ainda, com prejuízos de mobilidade ocasionados pela idade ou por outros fatores. Com isso esperamos contribuir para novas leituras sobre o Tai Chi Chuan, prática que, como pontuou Zibin Guo (2012), é uma intervenção acessível, de baixo custo e segura para praticantes com alguma deficiência motora.

\section{MÉTOdo}

Realizamos uma revisão da literatura em português e inglês com as seguintes palavraschave: "seated tai chi" OR "sitting tai chi” OR "wheelchair tai chi” OR "adapted tai chi" e, em português, "tai chi" + "cadeira" OU "tai chi" + "sentado" OR "tai chi" + "adaptado". Foram utilizadas as seguintes base de dados: PubMed, BIREME, Scielo, Science Direct e Google Schoolar. Nelas, encontramos 1496 artigos, dos quais 17 entraram no critério de inclusão. Selecionamos trabalhos realizados de janeiro de 2007 até janeiro de 2017. 
Para este estudo, utilizamos os seguintes critérios de inclusão: (1) artigos em inglês e português; (2) trabalhos originais; (3) estudos com métodos de Tai Chi Chuan sentado e/ou adaptado com participantes com ou sem deficiência. Como critério de exclusão, utilizamos: (1) estudos associando a prática do Tai Chi Chuan adaptado e ação medicamentosa; (2) resumos publicados em anais de eventos acadêmicos; (3) artigos que não abordavam a modalidade estudada.

\section{TERMOS DA BUSCA}

"seated tai chi" OR "sitting tai chi" OR "wheelchair tai chi" OR "adapted tai chi"/ "tai chi" + "cadeira" OU "tai chi" + "sentado" OR "tai chi" + "adaptado"

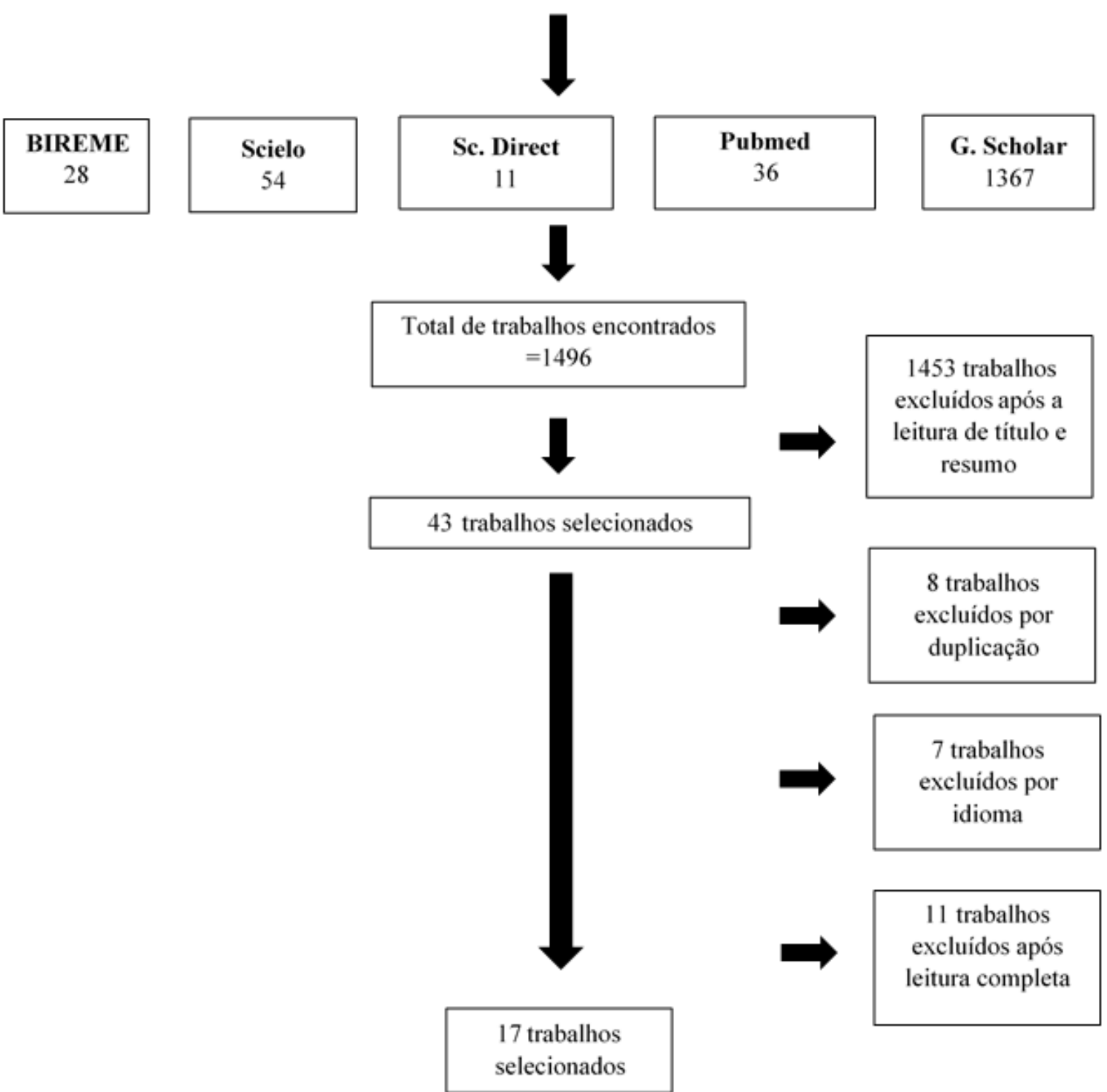

Figura 1 - Processo de seleção de artigos nas bases de dados. 


\section{Resultados E Discussão}

O objetivo do nosso estudo foi realizar uma revisão de literatura sobre trabalhos científicos envolvendo a prática do Tai Chi Chuan em posição sentada como prática corporal acessível. Com isso em vista, os trabalhos foram divididos em quatro categorias: Abordagens Político-práticas; Estudos Cinemáticos; Aspectos Físicos; e Aspectos Psicológicos. O Quadro 1 apresenta os trabalhos incluídos para análise.

Quadro 1 - Trabalhos incluídos na análise de resultados

\begin{tabular}{|c|c|c|c|}
\hline AUTOR(ES) & ANO & PÁIS & TÍTULO \\
\hline CHEUNG et al. & 2007 & Hong Kong & $\begin{array}{l}\text { Physical benefits of Tai Chi Chuan for individuals with } \\
\text { lower limb disabilities }\end{array}$ \\
\hline $\begin{array}{l}\text { LEUNG; } \\
\text { TSANG }\end{array}$ & 2008 & Hong Kong & $\begin{array}{l}\text { Comparison of the kinetic characteristics of standing and } \\
\text { sitting Tai Chi forms }\end{array}$ \\
\hline GUO; ZANG & 2010 & EUA & $\begin{array}{l}\text { Dancing in the Chair: Wheelchair Taijiquan for People } \\
\text { with Ambulatory Impairment }\end{array}$ \\
\hline $\mathrm{HO}$ et al. & 2011 & TAIWAN & $\begin{array}{l}\text { Analysis of Tai Chi as a Functional Rehabilitation } \\
\text { Treatment for Upper Extremities of Wheelchair Patients }\end{array}$ \\
\hline SIU; LUM & 2011 & Hong Kong & $\begin{array}{l}\text { Effects of sitting Tai Chi on sitting balance and self-care } \\
\text { ability in post-stroke older adults: a pilot study. }\end{array}$ \\
\hline LEE et al. & 2011 & Hong Kong & Kinematics and Energy Expenditure of Sitting T'ai Chi \\
\hline GUO & 2012 & EUA & $\begin{array}{l}\text { Thirteen postures of wheelchair Taijiquan (Tai Chi): } \\
\text { wheelchair use as an instrument of empowerment }\end{array}$ \\
\hline YU et al. & 2012 & Taiwan & $\begin{array}{l}\text { The immediate effect of wheelchair Tai Chi on autonomic } \\
\text { nervous modulation in subjects with chronic spinal cord } \\
\text { injury: A pilot study. }\end{array}$ \\
\hline COSTA et al. & 2013 & Brasil & $\begin{array}{l}\text { Interferência da Prática de Tai Chi Chuan na Saúde } \\
\text { Mental de Pessoas Com Lesão da Medula Espinhal. }\end{array}$ \\
\hline GEIB et al. & 2014 & EUA & $\begin{array}{l}\text { Using posturography to measure balance control during } \\
\text { seated tai chi. }\end{array}$ \\
\hline WANG et al. & 2015 & EUA & $\begin{array}{l}\text { Wheelchair Tai Chi as a Therapeutic Exercise for } \\
\text { Individuals with Spinal Cord Injury }\end{array}$ \\
\hline LEE et al. & 2015 & Hong Kong & $\begin{array}{l}\text { The effects of practicing sitting Tai Chi on balance } \\
\text { control and eye-hand coordination in the older adults: a } \\
\text { randomized controlled trial. }\end{array}$ \\
\hline TSANG et al. & 2015 & Hong Kong & $\begin{array}{l}\text { Sitting tai chi improves the balance control and muscle } \\
\text { strength of community-dwelling persons with spinal cord } \\
\text { injuries: a pilot study. }\end{array}$ \\
\hline SHEM et al. & 2016 & EUA & $\begin{array}{l}\text { Seated Tai Chi to alleviate pain and improve quality of } \\
\text { life in individuals with spinal cord disorder }\end{array}$ \\
\hline WANG et al. & 2016 & EUA & $\begin{array}{l}\text { Effects of wheelchair Tai Chi on physical and mental } \\
\text { health among elderly with disability. }\end{array}$ \\
\hline HSU et al. & 2016 & Austrália & $\begin{array}{l}\text { Seated Tai Chi versus usual activities in older people } \\
\text { using wheelchairs: A randomized controlled trial- }\end{array}$ \\
\hline PAN et al. & 2017 & Canada & $\begin{array}{l}\text { Adapting Tai Chi for Upper Limb Rehabilitation Post } \\
\text { Stroke: A Feasibility Study }\end{array}$ \\
\hline
\end{tabular}


O Quadro 2 apresenta os autores dos trabalhos separados por categorias. O trabalho de Wang et al. (2016) foi incluído em duas delas, por envolver a análise de variáveis tanto físicas como psicológicas.

Quadro 2 - Divisão dos autores dos trabalhos por categorias

\begin{tabular}{|l|l|l|l|}
\hline \multicolumn{1}{|c|}{$\begin{array}{c}\text { Estudos } \\
\text { Cinemáticos }\end{array}$} & $\begin{array}{c}\text { Abordagens político- } \\
\text { práticas }\end{array}$ & \multicolumn{1}{|c|}{$\begin{array}{c}\text { Aspectos } \\
\text { físicos }\end{array}$} & \multicolumn{1}{c|}{$\begin{array}{c}\text { Aspectos } \\
\text { psicológicos }\end{array}$} \\
\hline LEUNG; TSANG, 2008 & GUO; ZANG, 2010 & CHEUNG et al. 2007 & COSTA et al. 2013 \\
\hline HO et al., 2011 & GUO, 2012 & SIU; LUM, 2011 & TSANG et al. 2015 \\
\hline LEE et al., 2011 & WANG et al., 2015 & YU et al. 2012 & HSU et al. 2016 \\
\hline GEIB et al., 2014 & & TSANG, et al. 2015 & SHEM et al. 2016 \\
\hline LEE et al., 2015 & & WANG et al. 2016 & WANG et al. 2016 \\
\hline & & PAN et al., 2017 & \\
\hline
\end{tabular}

\section{ESTUDOS CINEMÁTICOS}

Leung e Tsang (2008) avaliaram a execução do Tai Chi por uma mestra de 57 anos, comparativamente na posição de pé e na posição sentada. Utilizando plataformas de pressão, concluíram que diferentes movimentos dentro da coreografia produzem graus distintos de deslocamento dos centros de pressão nas plantas dos pés, tendo a forma sentada produzido deslocamentos significativamente menores do que a forma em pé em todos os trechos da execução. Os autores sugerem que o Tai Chi em posição sentada pode ser um recurso terapêutico para reabilitação do equilíbrio em pacientes fragilizados, principalmente idosos que possuam dificuldade ficar em pé. Entretanto, a hipótese do estudo se limita a pacientes com controle motor nos membros, excluindo da análise, por exemplo, pacientes com paraplegias severas.

Já Ho et al. (2011) utilizaram um movimento de Tai Chi para avaliar comparativamente a amplitude de movimento e a velocidade angular de pico das articulações dos membros superiores entre pessoas sem deficiência e pessoas com deficiência. $\mathrm{O}$ grupo clínico, composto por 8 homens com deficiência nos membros inferiores e usuários de cadeira de rodas, apresentou graus significativamente menores $(\mathrm{p}<0.05)$ em praticamente todas as variáveis, à exceção da amplitude do movimento dos cotovelos. Os autores se basearam nesse resultado para implicar que a prática de Tai Chi Chuan poderia beneficiar a reabilitação e o desenvolvimento de amplitude nos membros superiores de pessoas com deficiência nos membros inferiores. Entretanto, essa parece ser uma suposição que parte de considerações vindas de outras pesquisas, e não propriamente de um caráter de aplicação experimental do Tai Chi. Os autores utilizam-no como ferramenta de análise e não como intervenção terapêutica.

A pesquisa de Lee et al. (2011) fez uma avaliação do deslocamento do centro de massa 
e traçou a estimativa de gasto energético em uma forma de Tai Chi desenvolvida para o estudo. A sequência foi feita por uma mestra experiente, em quatro condições: sentada, sentada e com cargas leves nas extremidades dos membros, de pé sem movimentação de membros inferiores, de pé com movimentação de membros inferiores.

As formas sentadas apresentaram menor intensidade, sendo a versão sem carga a de menor gasto energético. As formas em pé apresentaram maior gasto, notadamente a forma convencional com movimentos de membros inferiores. Quanto ao deslocamento do centro de massa, foi observado que a forma sentada, embora apresentando níveis de movimentação menores, ainda promove estímulos que exigem ajustes da postura corporal para a manutenção do equilíbrio. Como conclusão, os autores apontaram que há possibilidades de variação na prática do Tai Chi para indivíduos com diferentes graus de fragilidade ou limitação nos movimentos, de forma a modular os estímulos e promover adaptações através da intervenção.

O estudo piloto de Geib et al. (2014) investigou a qualidade de movimento de Tai Chi Chuan na posição sentada entre experientes e não experientes. Os autores utilizaram um software para avaliar a postura dos praticantes em posição sentada. Foram avaliadas variações no centro de pressão, utilizado como parâmetro de equilíbrio, além de quatro gestos técnicos, de forma separada e em seguida de forma sequencial. Os autores concluíram que a posturografia é um método útil para diferenciar a qualidade de movimento quando executado em posição sentada entre experientes e não experientes.

O estudo de Lee et al. (2015) teve como objetivo analisar os efeitos da prática no equilíbrio e na coordenação de movimento entre olho e mão, indicativo de coordenação apurada. Para isso, foram convidados 59 participantes idosos, que foram divididos entre grupo controle $(n=30)$ e grupo experimental $(n=29)$. O treinamento estimulou a mudança do centro de massa, mesmo em posição sentada. A ênfase nos movimentos para membros superiores explica a melhora da coordenação olho-mão. Assim, o estudo mostrou que o Tai Chi Chuan na posição sentada pode ser uma opção de terapia para idosos.

\section{ABORDAGENS POLÍTICO-PRÁTICAS}

Guo e Zang (2008) descrevem o processo pelo qual o Tai Chi em cadeira de rodas se estabeleceu na China entre 2005 e 2008, obtendo sucesso como programa de atividade física para pessoas com deficiência. Segundo os autores, isso se deu porque a prática, além de barata e acessível, possuía um grau de valorização cultural generalizado na sociedade chinesa. As técnicas são facilmente adaptáveis em termos de ritmo e amplitude, de modo que diferentes graus de prejuízo motor podem ser contemplados nos grupos.

No mesmo sentido Guo (2012) faz um outro relato, ainda sobre programas de Tai Chi Chuan em cadeira de rodas na China. Este trabalho resultou na criação da forma de 13 movimentos, apresentada por 50 praticantes no Olympics/Paralympics Cultural Festival no ano de 2008 em Beijing. O autor destaca que essa forma adaptada de Tai Chi não leva tanto em conta aspectos marciais, típicos das formas tradicionais, mas privilegia a mobilidade dos 
membros superiores, da coluna lombar e dos quadris, quando possível. Guo (2012) aponta que o Tai Chi Chuan em cadeira de rodas pode ser considerado acessível, barato e seguro, além de promover um sentimento de empoderamento aos praticantes pela assimilação da cadeira como um elemento de movimentação na coreografia.

Já Wang et al. (2015) propuseram a criação de uma forma de Tai Chi Chuan de 10 movimentos, específicos para pessoas com comprometimento de membros inferiores e/ou usuários de cadeira de rodas, chamado WTC-10 - Wheelchair TaiChi 10 movimentos. O instrumento é composto por 2 sessões, com 5 movimentos cada, descritos detalhadamente pelos autores. Os autores apresentam a ordem correta de aprendizado da forma, bem como ilustrações. Wang et al. (2015) ressaltam que, apesar do WTC-10 ter sido criado para pessoas com lesão medular, a prática pode ser incentivada para pessoas com outros comprometimentos. Assim, a produção do material explicativo é uma forma de difusão do conhecimento que pode ser útil para a população em geral.

Esses estudos contribuem para a difusão e prática de Tai Chi Chuan adaptado em diferentes contextos, seja pela criação de métodos que englobem um público historicamente negligenciado, ou pela criação de técnica de execução do Tai Chi Chuan adaptado de forma sistematizada e acessível.

\section{AsPectos Físicos}

Cheung et al., (2007) convidaram 22 pessoas com comprometimento de membros inferiores, sendo 11 usuários de cadeira de rodas e 11 cuja marcha é feita com auxílio de equipamento (grupo experimental); e 17 pessoas do grupo controle, com comprometimento semelhante ao grupo experimental (10 usuários de cadeira de rodas e 7 utilizam aparelhos para andar). Foram avaliadas em ambos os grupos a função cardiovascular e pulmonar, além da amplitude de movimento do ombro.

O grupo experimental apresentou melhora na amplitude de movimento do ombro: rotação externa do ombro direito, extensão do ombro direito; extensão do ombro esquerdo. Para as variáveis de função cardíaca e pulmonar, abdução do ombro direito e esquerdo e rotação externa do ombro esquerdo não houve diferenças estatisticamente significativas. Os autores concluíram que o Tai Chi melhora a mobilidade do ombro e, além disso, indicaram uma possível associação com ganhos de flexibilidade e força para os músculos dessa região.

Siu e Lum (2011) submeteram um grupo de 19 idosos com dificuldade de locomoção a um programa de treino de Tai Chi em posição sentada. Todos os indivíduos apresentavam hemiparesia em decorrência de acidente vascular cerebral, além de dificuldade ou incapacidade de permanecer em pé. Os efeitos da intervenção foram mensurados a partir da variável obtida pelo teste de "sentar e alcançar" feito na cadeira. Os resultados foram negativos, com $\mathrm{p}=0.028 \mathrm{em}$ um sentido não funcional. Os autores concluíram que o Tai Chi em posição sentada não é uma intervenção proveitosa para idosos com mobilidade prejudicada por quadro de AVC. 
Yu et al. (2012), por sua vez, estudaram os efeitos da prática sobre a modulação do sistema nervoso autonômico em 10 sujeitos com lesão medular, dentre os quais cinco eram tetraplégicos (lesão entre C3 e C6) e cinco paraplégicos (lesão entre T4 e T12). Foi mensurada a variação dos sinais cardíacos dos participantes através do ecocardiograma para verificar as alterações nos sinais do sistema nervoso. Neste estudo, as medidas avaliadas não tiveram diferença estatisticamente significativa para idade e tempo pós lesão para os sujeitos avaliados. Além disso, constatou-se que houve melhora significativa nas atividades simpáticas (diminuição do pico de frequência máxima) e melhora do balanço simpato-vagal, mensurado através da divisão entre a área de baixa frequência (entre 0 e 0,04Hz) e área de alta frequência (entre 0,15 e $0,4 \mathrm{~Hz}$ ) para o ecocardiograma. Como limitações do estudo, os autores apontaram: (1) pequena quantidade de sujeitos $(\mathrm{n}=10)$; (2) falta de um grupo controle, o que tornam limitados os resultados dessa pesquisa para a prescrição da prática a lesados medulares; (3) presença de tetraplégicos e paraplégicos no mesmo grupo, o que torna o grupo pouco homogêneo e, por conseguinte, os resultados pouco precisos; (4) para os autores, a utilização de equipamentos com melhor capacidade de armazenamento poderia influenciar nos resultados e (5) sugerem que estudos futuros estudem o efeito de longo prazo do Tai Chi Sentado nas variáveis de pressão arterial e padrão respiratório.

Similar ao trabalho anterior, Tsang et al. (2015) submeteram 11 sujeitos com lesão na medula espinhal a uma intervenção com Tai Chi Chuan. Foram avaliados a força muscular, através do teste da preensão manual, e o controle do equilíbrio na posição sentada, através de uma plataforma de força e de um protocolo para medição de deslocamentos no centro de pressão. Neste estudo, houve um grupo controle $(n=8)$, porém os autores apontam algumas limitações como a heterogeneidade dos participantes (gênero, idade, nível da lesão) e o fato da distribuição entre grupo experimental e controle não ter sido feita por sorteio. De qualquer modo, foi possível concluir que o treino de Tai Chi Chuan na posição sentada influenciou positivamente no controle do equilíbrio e força muscular geral, expressos em melhores desempenhos no teste de preensão manual e no protocolo de deslocamento do peso.

Wang et al. (2016) realizaram um estudo avaliando os efeitos da prática da forma de 10 movimentos de Tai Chi Chuan na posição sentada com indivíduos idosos. Foram observados parâmetros de sinais vitais (frequência cardíaca, pressão sistólica e diastólica), amplitude de movimento, alcance ao sentar e dor. Este estudo contou com 40 sujeitos, divididos em grupo controle $(n=20)$ e grupo experimental $(n=20)$. O estudo mostrou resultados positivos na pressão sistólica e diastólica, cujos mecanismos exatos os autores não apontam, mas relacionam com o caráter meditativo da prática. Mostrou ainda melhoras na amplitude de movimento para rotação de ombro e tronco e melhora do nível de atividade física, além de ser considerada uma prática segura para idosos.

Pan et al. (2017) analisaram os efeitos da prática de Tai Chi na reabilitação de membros superiores afetados por derrame. O estudo submeteu 11 pacientes com paresia persistente aos treinos. A intervenção mostrou-se possível em diferentes condições, em posição sentada, posição estática em pé ou com a movimentação convencional. Os autores sugerem o Tai Chi Chuan como uma opção viável para reabilitação de pacientes em situação 
pós-derrame devido à sua adaptabilidade. Adicionalmente, destacam que a intervenção diminuiu os níveis de dor no ombro daqueles que as possuíam.

\section{Aspectos Psicológicos}

No estudo de Costa et al. (2013) um grupo de 8 pessoas com lesão medular foi submetido a intervenções de Tai Chi Chuan associadas com tratamento fisioterápico usual. Em comparação com o grupo controle, que foi submetido apenas à fisioterapia, o grupo experimental apresentou scores significativamente melhores no Inventário de Depressão de Beck ( $\mathrm{p}=0.021)$ e na Escala de Auto Estima de Rosenberg $(\mathrm{p}=0.015)$. Além disso, os praticantes relataram melhoras subjetivas em aspectos psicológicos, sociais e físicos. As autoras do artigo sugerem, dessa forma, que a prática do Tai Chi associada à fisioterapia produz melhoras importantes nos fatores psicossociais ligados à reabilitação de pessoas com lesão medular.

Em Shem et al. (2016) 26 pessoas com lesão medular participaram de um programa de Tai Chi Chuan em posição sentada. Dentre os sujeitos, apenas 9 completaram todas as sessões e responderam os questionários do protocolo de pesquisa, visando avaliar as escalas pré-aula e pós-aula das variáveis dor, sensação emocional de bem-estar, distração mental, sensação física de bem-estar e senso de conexão espiritual. Todos apresentaram melhorias nessas variáveis no momento pós-aula. Os autores concluíram que o Tai Chi em posição sentada oferece benefícios em curto prazo que contribuem para a melhora da qualidade de vida nos lesionados medulares.

Wang et al. (2016) trabalharam com grupo controle $(n=20)$ e grupo experimental $(n=20)$ com Tai Chi Chuan sentado. Neste estudo, foram avaliados os aspectos psicológicos de autoeficácia e a subescala de saúde mental do instrumento SF-36, que engloba aspectos sociais, emoções, vitalidade, saúde mental e a combinação dos aspectos mentais. O protocolo de Tai Chi Chuan sentado melhorou aspectos da autoeficácia nos praticantes do grupo experimental comparado ao grupo controle. O protocolo mostrou melhoras para vitalidade e saúde mental.

Já o trabalho de Hsu et al. (2016) teve o objetivo de avaliar o impacto da prática em sintomas depressivos e qualidade de vida em idosos. O estudo contou com 60 sujeitos divididos em grupo controle e experimental. O grupo controle realizou atividades como karaokê, ouvir música e alongamento.

Para este estudo foi utilizado o questionário curto de depressão geriátrica (QCDG) e o instrumento de qualidade de vida da Organização Mundial da Saúde, versão breve (WHOQOL). Além disso, foi utilizado o Mini Exame do Estado Mental (MEEM) para avaliar a função cognitiva. O estudo mostrou que a prática de Tai Chi Chuan adaptado auxiliou na diminuição dos sintomas depressivos em cinco vezes, comparado com o grupo controle. Ademais, a qualidade de vida mostrou-se melhor no grupo experimental em todos os domínios. 
Por fim, o já citado trabalho de Tsang et al. (2015), além dos aspectos físicos já explanados, analisou o aspecto da qualidade de vida através do instrumento WHOQOL breve. Embora as outras variáveis analisadas tenham demonstrado resultados positivos, em relação à qualidade de vida os autores não observaram melhorar significativas, o que contraria os resultados de Hsu et al. (2016).

\section{CONSIDERAÇÕES FINAIS}

A revisão demonstrou que estudos relacionados a formas adaptadas de Tai Chi Chuan ainda são escassos em língua inglesa e portuguesa. Quase metade dos artigos tem origem em países asiáticos, como Hong Kong e Taiwan, o que nos sugere que pode haver, em idiomas dessa região, ainda mais produções científicas do que as que conseguimos acessar.

De forma geral, os estudos não apresentam uma descrição detalhada dos critérios para se considerar a execução de determinado movimento como dado válido para a pesquisa. Nesse sentido, o uso de imagens poderia ter contribuído para se indicar o padrão aceito de movimento para cada estudo, o que ajudaria, por conseguinte, a garantir o princípio da reprodutibilidade do conhecimento científico pela aplicação dos mesmos padrões de movimento em outras pesquisas. Tampouco se vê uma preocupação com a categorização do Tai Chi utilizado na intervenção - se é moderno ou tradicional, por exemplo - o que também pode trazer prejuízos à precisão na divulgação do método, produzindo igualmente problemas de reprodutibilidade.

As imprecisões em algumas dessas pesquisas seguem, assim, uma tendência frequente nas produções envolvendo estudos clínicos com Tai Chi Chuan convencional (YANG et al., 2015). As pesquisas com tal perfil nesta revisão tenderam a utilizar, também, amostras populacionais reduzidas, reflexo das próprias dificuldades sociais e logísticas de adesão e permanência de pessoas com deficiência a programas de exercícios físicos (GUO, 2010). No caso do estudo de Yu et al. (2012), por exemplo, soma-se a esses problemas a ausência de um grupo controle, o que é indispensável para se determinar se os resultados decorreram da intervenção ou de outros fatores não controlados.

Além das dificuldades citadas, cabe frisar que praticamente todas as produções levantadas enquadram a prática no paradigma tradicionalmente adotado, qual seja, o terapêutico, ligado à saúde e à reabilitação. Nenhuma das pesquisas abordou o Tai Chi Chuan em posição sentada dentro de aspectos marciais ou esportivos, que são presentes no espectro da prática não adaptada.

De modo geral, os estudos de cunho político-prático caracterizaram a modalidade em posição sentada como sendo uma prática de baixo custo e acessível, com gestual facilmente adaptável em termos de ritmo e amplitude. Privilegiando a mobilidade e não a marcialidade, a prática contemplaria diferentes graus de prejuízo motor. Ajudaria a promover, dessa forma, 
um sentimento de empoderamento a um grupo excluído. Para usuários de cadeira de rodas, poderia ainda favorecer a construção de uma outra relação com a cadeira, assimilando-a na coreografia.

Quanto aos estudos ligados à análise cinemática, podemos afirmar que o indicativo geral que oferecem é o de que a prática do Tai Chi em posição sentada, embora menos intensa que a convencional, ainda promove deslocamentos do centro de massa e, consequentemente, dos centros de pressão nos pontos de apoio (pés em relação ao chão ou, na ausência de controle motor, os apoios do corpo na cadeira). Nesse sentido, indica-se, portanto, que os indivíduos realizam ajustes posturais na prática adaptada que podem, segundo as pesquisas abordadas, contribuir para a melhora do equilíbrio.

Por fim, os artigos analisados indicam também, embora com certa heterogeneidade quanto aos procedimentos metodológicos, que o Tai Chi Chuan em posição sentada pode gerar efeitos positivos principalmente sobre a amplitude dos movimentos de ombros, com possíveis ganhos de força e flexibilidade na musculatura correspondente. Benefícios também foram observados na força de preensão manual e no controle de equilíbrio. Quanto às funções cardíacas e pulmonares, o estudo de Cheung et al. (2007) não obteve qualquer confirmação de benefício, embora as pesquisas de Yu et al. (2012) e de Wang et al. (2016) tenham indicado melhoras nos sinais cardíacos e na pressão arterial, respectivamente.

Nos aspectos psicológicos, o principal enfoque foi em pessoas com lesão medular, e todas as pesquisas abordadas apresentaram resultados significativamente positivos sobre parâmetros variados, como autoestima, autoeficácia, qualidade de vida e saúde mental, além de outros critérios mais subjetivos, obtidos por declaração dos sujeitos dos grupos experimentais. Houve também indicações de melhoras nos sintomas depressivos. Apenas a pesquisa de Tsang et al. (2016) não apresentou diferenças nos resultados pós intervenção, especificamente no parâmetro qualidade de vida.

Como sugestão, apontamos para a necessidade de se estabelecer um rigor maior na aplicação de protocolos, o que incluiria determinar o estilo do Tai Chi Chuan e os movimentos utilizados, assim como delimitar os critérios de inclusão de sujeitos com base na aproximação de seus movimentos com uma técnica considerada ideal. Com isso, não se visaria tornar a prática estática, mas apenas garantir que as pesquisas tenham um grau de similaridade que as tornem comparáveis, permitindo a reprodutibilidade do conhecimento científico em Tai Chi Chuan sentado e a qualidade das revisões ulteriores.

Ressaltamos também a necessidade da existência de grupos controle em estudos sobre saúde, qualidade de vida e aspectos psicológicos, sem os quais não se pode dizer se os benefícios mensurados devem-se à intervenção experimental ou a fatores externos. Uma maior precisão na descrição dos mecanismos fisiológicos envolvidos nos resultados das intervenções experimentais é necessária. Números amostrais maiores devem também ser buscados, pois quanto maior a população estudada, mais os resultados podem ser atribuídos à prática estudada e menos às variantes individuais. A inflação do $n$ amostral poderia ser 
conseguida, por exemplo, com projetos de extensão universitária de longo prazo.

Por fim, esse objeto de pesquisa precisa ser também apreciado sob outros enfoques que não apenas as medidas relacionadas à saúde e a qualidade de vida. O Tai Chi Chuan adaptado não é apenas mecânica, com influência em fatores psicológicos e fisiológicos - ele é uma prática, um ato que possui várias dimensões. Portanto, mais pesquisas poderiam ser promovidas incluindo sua dimensão marcial, técnica, esportiva, recreativa, pedagógica, social, cultural e política.

\section{REFERÊNCIAS}

ANTUNES, Marcelo Moreira. Artes marciais para pessoas com deficiência. Rio de Janeiro: Multifoco, 2016.

BROADHEAD, Geoff. Guest editorial: how APAQ was born? Adapted Physical Activity Quarterly, Birmingham, v. 34, n. 3, p. 209-216, 2017.

CHEUNG, Siu Yin et al. Physical benefits of Tai Chi Chuan for individuals with lower limb disabilities. Occupational therapy international, v. 14, n. 1, p. 1-10, 2007.

COSTA, Viviane de Souza Pinho et al. Interferência da Prática de Tai Chi Chuan na Saúde Mental de Pessoas Com Lesão da Medula Espinhal. Journal of Health Sciences, Londrina, v. 15, n. 4, p. 273-277, 2013.

DU, Shizheng et al. Taichi exercise for self-rated sleep quality in older people: a systematic review and meta-analysis. International journal of nursing studies, Southampton, v. 52, n. 1, p. 368-379, 2015.

FIGUEIREDO, Valéria Maria Chaves de; TAVARES, Maria da Consolação Gomes Cunha Fernandes; VENÂNCIO, Silvana. Olhar para o corpo que dança: um sentido para a pessoa portadora de deficiência visual. Movimento, Rio Grande do Sul, v. 5, n. 11, p. 65-73, 1999. Disponível em: <https://seer.ufrgs.br/Movimento/article/view/2487/1136>.

GEIB, Roy W. et al. Using posturography to measure balance control during seated tai chi. Biomedical sciences instrumentation, New York, v. 50, p. 235-241, 2014.

GOODWIN, Donna L.; KROHN, Joan; KUHNLE, Arvid. Beyond the wheelchair: the experience of dance. Adapted Physical Activity Quarterly, Birmingham, v. 21, n. 3, p. 229247, 2004.

GUIMARÃES, Juliano Silveira; DE LIMA, Alisson Padilha; CARDOSO, Fabrício Bruno. Efeito da prática do Tai Chi Chuan na autoestima e autoimagem de idosos. RBPFEX-Revista Brasileira de Prescrição e Fisiologia do Exercício, Maranhão, v. 11, n. 71, p. 952-959, 2018.

GUO, Zibin. Thirteen postures of wheelchair taijiquan (tai chi): wheelchair use as an instrument of empowerment. Technology \& Innovation, Tampa, v. 13, n. 4, p. 267-279, 2012. 
GUO, Zibin; ZANG, Yuyang. Dancing in the Chair: Wheelchair Taijiquan for People with Ambulatory Impairment. International Journal of Disability, Community \& Rehabilitation, v. 9, n. 1, p. 1, 2010.

HALL, Amanda et al. Effectiveness of tai chi for chronic musculoskeletal pain conditions: updated systematic review and meta-analysis. Physical therapy, Boston, v. 97, n. 2, p. 227238, 2017.

HSU, Chen-Yuan et al. Seated Tai Chi versus usual activities in older people using wheelchairs: A randomized controlled trial. Complementary therapies in medicine, v. 24, p. 16, feb. 2016. Disponível em:

<https://www.sciencedirect.com/science/article/pii/S0965229915300182?via\%3Dihub>.

KONG, Ling Jun et al. Tai chi for chronic pain conditions: a systematic review and metaanalysis of randomized controlled trials. Scientific reports, London, v. 6, p. 25325, apr. 2016. Disponível em: <https://www.ncbi.nlm.nih.gov/pmc/articles/PMC4850460/>.

LEE, Ken Y. T. et al. Kinematics and Energy Expenditure of Sitting T'ai Chi. Journal of alternative and complementary medicine, New York, v. 17, n. 8, p. 665-668, 2011. Disponivel em: 〈https://www.liebertpub.com/doi/pdf/10.1089/acm.2011.0147〉.

LEE, Ken Y. T.; HUI-CHAN, Christina W. Y.; TSANG, Willian W. N. The effects of practicing sitting Tai Chi on balance control and eye-hand coordination in the older adults: a randomized controlled trial. Desability and rehabilitation, v. 37, n. 9, p.790-4, 2015.

LEE, Myeong Soo et al. A systematic review and meta-analysis of tai chi for treating type 2 diabetes. Maturitas, Oxford, v. 80, n. 1, p. 14-23, 2015.

LEUNG, Eric S. F.; TSANG, Willian W. N. Comparison of the kinetic characteristics of standing and sitting Tai Chi forms. Disability and rehabilitation, v. 30, n. 25, p. 1891-900, 2008.

MARINHO, Marina Santos et al. Efeitos do Tai Chi Chuan na incidência de quedas, no medo de cair e no equilíbrio em idosos: uma revisão sistemática de ensaios clínicos aleatorizados. Revista Brasileira de Geriatria e Gerontologia, Rio de Janeiro, v. 10, n. 2, p. 243-256, 2007.

MARTIN, Jeffrey; WHALEN, Lauren. Effective practices of coaching disability sport. European Journal of Adapted Physical Activity, Olomouc, v. 7, n. 2, p. 13-23, 2014.

MELO, Flávio Anderson Pedrosa de; FUMES, Neiza de Lourdes Frederico. O esporte adaptado no município de Maceió/AL: des/caminhos traçados pelas políticas públicas. Revista da Sobama, Marília, v. 14, n. 2, p. 41-48, 2013.

MUNSTER, Mey de Abreu van; ALMEIDA, José Júlio Gavião de. Esporte, lazer e deficiência: avanços e retrocessos no município de São Carlos. Revista da Sobama, Marília, v. 17, n. 1, p. 19-24, 2016.

NAFAPA - North American Federation of Adapted Physical Activity. Promotion, Dissemination, and Practical Application of Results \& Findings in the Field of Physical 
Activity for Populations with Disabilities and/or Special Needs. 2016. Disponível em: <https://www.nafapa.net/nafapa-2016-symposium>. Acesso em: 14 mar. 2018.

NERY, Rosane Maria et al. Tai Chi Chuan como reabilitação cardíaca na doença arterial coronária: revisão sistemática. Arquivos brasileiros de cardiologia, São Paulo, v. 102, n. 6, p. 588-592, 2014.

PALMA, Luciana Erina et al. Ensino da capoeira para pessoas com deficiência intelectual. Revista da Sobama, Marília, v. 13, n. 1, p. 27-30, 2012.

PAN, Shujuan et al. Adapting Tai Chi for Upper Limb Rehabilitation Post Stroke: A Feasibility Study. Medicines (Basel), Basel, v. 4, n. 4, p. 71, 2017.

PAN, Yuanqing et al. Tai chi chuan exercise for patients with breast cancer: a systematic review and meta-analysis. Evidence-Based Complementary and Alternative Medicine, London, v. 2015, 2015.

ROSSI, Patricia; MUNSTER, Mey de Abreu van. Dança e deficiência: uma revisão bibliográfica em teses e dissertações nacionais. Movimento, Rio Grande do Sul, v. 19, n. 4, p. 181-205, 2013.

ROSWAL, Peggy M.; SHERRILL, Claudine; ROSWAL, Glenn M. A comparison of data based and creative dance pedagogies in teaching mentally retarded youth. Adapted Physical Activity Quarterly, Birmingham, v. 5, n. 3, p. 212-222, 1988.

SANTOS, Romário Silva et al. Acessibilidade de cadeirantes em um equipamento específico de lazer: o estádio de futebol Bastião na cidade de Aracaju/SE. LICERE - Revista do Programa de Pós-graduação Interdisciplinar em Estudos do Lazer, Belo Horizonte, v. 20, n. 3, p. 289-312, 2017.

SHEM, Kazuko et al. Seated Tai Chi to alleviate pain and improve quality of life in individuals with spinal cord disorder. The journal of spinal cord medicine, Springfield, v. 39, n. 3, p. 353-8, 2016.

SILVA, Cláudio Silvério da; NETO, Samuel de Souza; DRIGO, Alexandre Janotta. A Educação Física Adaptada nos registros da Revista da Sociedade Brasileira de Atividade Motora Adaptada no período de 1996 a 2007. Revista da Sobama, Marília, v. 13, n. 1, p. 1-6, 2012.

SILVA, Junior Vagner Pereira da et al. Parque Itanhangá e acessibilidade às pessoas com deficiência física e visual. LICERE - Revista do Programa de Pós-graduação Interdisciplinar em Estudos do Lazer, Belo Horizonte, v. 16, n. 1, 2013.

SIU, Liu H.; LUM, Christopher. Effects of sitting Tai Chi on sitting balance and self-care ability in post-stroke older adults: a pilot study. Desability and rehabilitation, v. 37, n. 9, p. 790-794, 2015.

SOUZA, Joslei Viana de. Editorial. Revista da Sociedade Brasileira de Atividade Motora Adaptada, Marília, v. 13, n. 2, suplemento, 2012. 
TSANG, William W. N. et al. Sitting tai chi improves the balance control and muscle strength of community-dwelling persons with spinal cord injuries: a pilot study. Evidence-based complementary and alternative medicine, v. 2015, p. 1-9, 2015.

WANG, Fang et al. The effects of tai chi on depression, anxiety, and psychological wellbeing: a systematic review and meta-analysis. International journal of behavioral medicine, Marburg, v. 21, n. 4, p. 605-617, 2014.

WANG, Yong Tai et al. Wheelchair Tai Chi as a Therapeutic Exercise for Individuals with Spinal Cord Injury. Journal of Physical Education, Recreation \& Dance, Reston (USA), v. 86 , n. 5, p. 27-37, 2015.

WANG, Yong Tai et al. Effects of wheelchair Tai Chi on physical and mental health among elderly with disability. Research in sports medicine, v. 24, n. 3, p. 157-170, 2016.

YANG, Guo-Yan et al. Evidence base of clinical studies on Tai Chi: a bibliometric analysis. PloS one, San Francisco, v. 10, n. 3, p. e0120655, 2015.

YOSHIDA, Hélio Mamoru; SANTOS NETO, Samuel Ribeiro dos. Wushu e aspectos do treinamento: uma revisão da literatura. Caderno de Educação Física e Esporte, Marechal Cândido Rondon, v. 14, n. 1, p. 69-78, 2016.

YU, Shang-Ming et al. The immediate effect of wheelchair Tai Chi on autonomic nervous modulation in subjects with chronic spinal cord injury: A pilot study. International SportMed Journal, Lausanne, v. 13, n. 3, p. 96-108, 2012.

ZHENG, Guohua et al. Tai chi chuan for the primary prevention of stroke in middle-aged and elderly adults: a systematic review. Evidence-Based Complementary and Alternative Medicine, London, v. 2015, 2015a.

ZHENG, Guohua et al. The effect of Tai Chi training on cardiorespiratory fitness in healthy adults: a systematic review and meta-analysis. PloS one, San Francisco, v. 10, n. 2, p. e0117360, 2015b.

ZHENG, Guohua et al. Tai chi and the protection of cognitive ability: a systematic review of prospective studies in healthy adults. American journal of preventive medicine, Ann Arbor, v. 49, n. 1, p. 89-97, 2015c. 